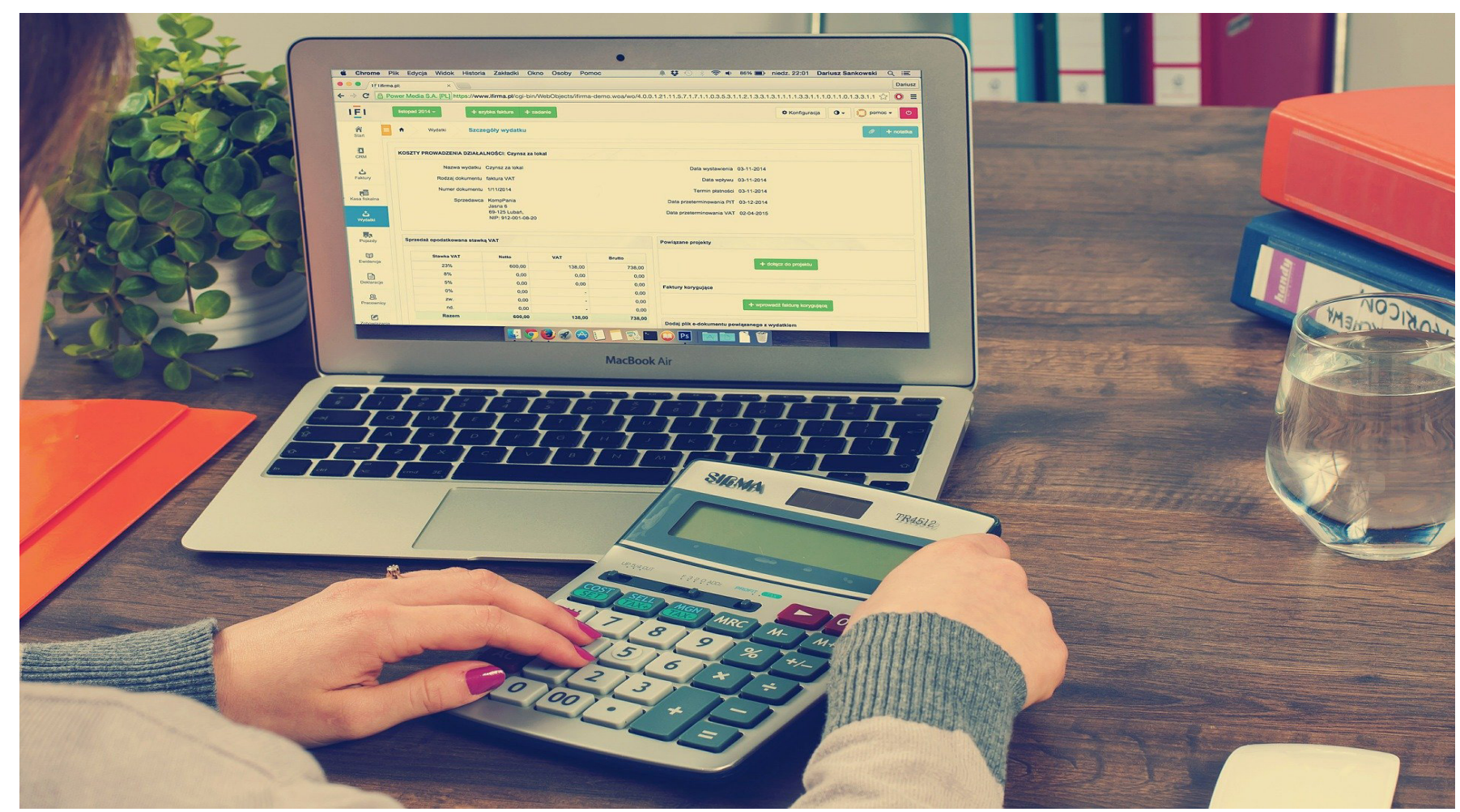

\title{
Relación entre el compromisoético del Contador Público y la importancia de esta labor ante la sociedad
}

\section{Relationship between the public accountant's ethical commitment and the importance of this work to society}

Yair Rolando Casadiego-Duque

Magíster en Planificación Global, yaircasadiego@ufps.edu.co,

Orcid: https://orcid.org/0000-0003-3649-8042, Universidad Francisco de Paula Santander, Cúcuta, Colombia

Carlos Fabián Rico-Rojas

Magíster Dirección Estratégica Especialidad Gerencia, carlosfabianrr@ufps.edu.co Orcid: https://orcid.org/0000-0002-0834-0339 Universidad Francisco de Paula Santander, Cúcuta, Colombia

\section{Viviana Paola Delgado-Sánchez}

Máster en Dirección y gestión financiera, vivianapaolads@ufps.edu.co, Orcid: https://orcid.org/0000-0003-1397-7526, Universidad Francisco de Paula Santander, Cúcuta, Colombia 


\section{Resumen}

El objetivo del presente articulo es analizar los aspectos que reflejan la relación entre el compromiso ético del contador público y la importancia que este puede llegar a tener en la sociedad, para lo cual se utilizó como método el anailisis documental, empezando por los conceptos básicos y llegando a temas coyunturales que afectan la situación del tema, también se mencionaron los principios y estatutos que rigen el compromiso ético de la profesión contable como lo es la ley 43 de 1990 cuyo contenido reglamenta el actuar del contador público en Colombia, en un desarrollo más a fondo podremos visualizar factores como la fe pública, que es sino uno de los baluartes de la profesión contable y además cobra mucha importancia debido a su validez absoluta sobre la información, el contador como actor social su relación directa, dado su responsabilidad, y no menos importante y que no debe desconocer, las diversas problemáticas que afectarían la ausencia del comportamiento ético, el impacto social que esto causaría y por ultimo posibles soluciones o alternativas para la problemática generada y que se pueda ver reflejado un mayor compromiso por parte del contador público y sea un impacto positivo para la sociedad.

Palabras clave: Comportamiento, Compromiso, Contador público, Ética, Sociedad.

\section{Abstract}

During the development of the following article we will analyze the aspects that reflect the relationship between the ethical commitment of the public accountant and the importance that this can have in society, starting with the basic concepts and reaching the conjunctural issues that affect the situation of the topic, We will also mention the principles and statutes governing the ethical commitment of the accounting profession, such as Law 43 of 1990, whose content regulates the actions of public accountants in Colombia, in a more indepth development we will be able to visualize factors such as public faith, which is but one of the bastions of our profession and also takes on great importance due to its absolute validity on information, the accountant as a social actor its direct relationship, given its responsibility, and no less important and that we must not ignore, the various problems that would affect ethical behavior, and could generate a risk under possible threats, the social impact that this would cause and finally possible solutions or alternatives to the problems generated and that can be reflected a greater commitment on the part of the accountant and be a positive impact on society.

Keywords: Behavior, commitment, public accountant, ethics, society. 
Introducción

A lo largo del tiempo ha observardo la constante evolución que ha logrado tener la Contaduría Pública, pasando de ser una actividad empírica, a ser reconocida como una de las profesiones más relevantes para el sistema económico y productivo del pais, siedo allí donde se genera el compromiso para con el cumplimiento de lo establecido a la ley, y formando así una profesión integral y oficial, de la cual dependen un sin número de asuntos que sin duda alguna son de suma importancia en términos globales y generales, es allí donde se genera el compromiso social para el Contador Público, la ContaduríaPública es una disciplina que en Colombia es conocida por el compromiso de velar por la correcta aplicación de la contabilidad en los diferentes entes empresariales, por lo tanto se podria decir que la profesión contable se ha convertido en el presente en un pilar fundamental para la toma de decisiones en base global lo cual la relaciona directamente con un impacto social, puesto a que la actividad contable se desempeña en dos áreas la pública y la privada, la formación del profesional contable debe ser impartidad desde la construcción de valores para poder actuar con responsabilidad, aquella que se le fue dada desde la ley 43 de 1990, donde mas que una profesión liberal es una profesión de carácter social, una profesión con una responsabilidad social sabiendo que de sus actuaciones se verán repercutidas en la sociedad hiendo en contra del bien común, esta responsabilidad social implica un comportamiento correcto a la hora de realizar el procesamiento de información para emitir estados financieros e informes sobre los aspectos relevantes de la estructura económica de la empresa.

Realizar este tipo de investigaciones son motivadas por la importancia que tiene en el pais esta profesión, es necesario realizarlo para analizar la relación existente ente la ética vista como los actos humanos que imparte el buen comportamiento y las buenas acciones de acuerdo con la importancia que tiene el impacto en la sociedad.

El profesional contable debe ser conciente en su actuar; debido a que es un acto que interesa al publico en el entorno empresarial en general, que sus decisiones afectan la economía de un pais y esto a su vez limita los recuersos para las personas menos favorecidas económicamente.

\section{Materiales y métodos}

En esta investigación se realizó con enfoque cualitativo, ya que lo que se realizó analizó los aspectos que reflejan la relación entre el compromiso ético del Contador Público y la importancia que este puede llegar a tener en la sociedad, el tipo de investigación utilizado fue descriptivo-explicativa, se analizó y determinó las causas del fenómeno en mención para asi dar opiniones que permitan interpretar la investigación.

\section{Resultados y discusión}

El compromiso de la ética del Contador Público ante la sociedad. ¿Quién es el contador público?

Se conoce al Contador Público como el profesional que se encarga de la organización de información contable en las entidades y dar fe pública de que estas cumplan con lo establecido en la ley. "Universalmente se conoce como Contador Público, aquel profesional dedicado a aplicar, analizar e interpretar la información contable y financiera de una organización" [1]. Todo esto con el fin de diseñar y proporcionar estrategias y medidas para la toma de decisiones en materia financiera. Es de suma importancia para la actividad del profesional contable actuar siempre bajo los principios éticos establecidos. 


\section{0}

"Se entiende por Contador Público la persona natural que, mediante la inscripción que acredite su competencia profesional en los términos de la presente Ley, está facultada para dar fe pública de hechos propios del ámbito de su profesión" [2]. Es el contador público uno de los profesionales más ligados al tema ético social, debido a su actividad el impacto que sus actos podrían causar en múltiples aspectos. Está sujeto a la vigilancia rigurosa de la junta central de contadores, la cual a su vez en la encargada de darle la facultad del ejercicio de la fe pública respaldada por la expedición y validez de su tarjeta profesional.

\section{Sociedad}

El termino sociedad proviene del latín "societas" y se refiere a una agrupación de seres vivos que habitan en comunidad, en un proceso de interacción en el cual se destacan aspectos como el equilibrio y la constante evolución, estableciendo relaciones entre los mismos individuos, por lo tanto, se puede considerar a una sociedad como un grupo de relaciones y costumbres.

\section{La ética}

La Ética es un conjunto de conocimientos sistemáticos, metódicos y racionales basados en la experiencia y fundados en principios [3], es una manera de actuar por parte del ser humano, basada en una serie de factores y parámetros que se establecen para regular el comportamiento dentro de una sociedad, las existencias de los principios de la ética siempre van a llevar a que la conducta vaya de acuerdo con dichos parámetros establecidos para mantener lo generalmente aceptado como lo correcto o lo más sensato.

"La ética tiene que ver con el compromiso de ser una buena persona, la cual pasa por la realización del bien común, es la rama del saber que se ocupa del estudio de las acciones morales de los individuos y de los grupos, así como de las reglas y normas que rigen ese comportamiento en una sociedad determinada" [4].

¿Qué es la ética profesional?

"La ética Profesional es reconocida como la ciencia normativa que estudia los deberes y los derechos de los profesionales en el cumplimiento de sus actividades Profesionales" [5], se puede catalogar la ética profesional como aquel conjunto principios que regulan la conducta de los seres humanos, por lo cual se siguen unos patrones de comportamiento ya determinados, es un concepto que podemos adaptar a cualquier ámbito, por ejemplo, el profesional en cualquier área debe seguir ciertos parámetros que lo llevaran a tener un comportamiento ético, "La ética profesional se refiere al conjunto de normas o principios por los cuales debe regirse una persona en el ámbito laboral" [6].

Esto lleva a definie la ética como un principio fundamental no solo en la formación, sino también en el desarrollo profesional, la ética es la garantía de la confiabilidad del actuar de las personas, para el Contador Público sin duda es notable el aspecto de regirse bajo los principios de la ética profesional debido a la importancia y delicadeza de sus facultades y todo lo que estas abarcan, por lo tanto, es un tema de vital importancia en el día a día del área contable.

\section{Ética del Contador}

Se entiende como ética del Contador, ese patrón de comportamiento que le indica según lo establecido y aceptado está actuando de la mejor manera, y por lo tanto cumpliendo plenamente con su deber, por tanto, se tiene en cuenta los comportamientos éticos como un compromiso fundamental casi como obligatorio para el buen desempeño de sus 
facultades y por la delicadeza de los asuntos tratados dentro de las mismas,

"El profesional ético comprende que sus principios y valores no son negociables, y esto, sin lugar a dudas, se constituye en el mayor activo que pueda poseer una compañía, y le blindará, ciertamente, para enfrentar los ataques de una dinámica social que cada día parece subestimar los comportamientos éticos" [2].

Relevancia de la conducta ética en la Contaduría Publica.

Como se resaltaba en el ámbito anterior la ética profesional es un factor fundamental en la formación y desarrollo del mismo, debido a que es la garantía de hacer las cosas de acuerdo a los principios de conducta generalmente aceptados, por lo cual no se puede desconocer que es el primer aspecto a tener en cuenta al momento del actuar, si las acciones individuales como profesional van de acuerdo con lo que mencionado anteriormente, se prodria relacionar los aspectos éticos del profesional con la disciplina puesto que son dos factores que suelen ir de la mano, por tanto se presume. "La importancia de la ética profesional, como parte del sistema de relación y disciplina, que es esencial en cualquier sociedad civilizada, estriba en que permite mantener la armonía, protegiendo a la sociedad de los actos irresponsables de una persona individual" [7].

Por lo cual se puede de nuevo resaltar que gracias a las conductas éticas se tiene la garantía de que el profesional realice su actividad y mantenga el equilibrio social, en el caso del Contador Público ya sabemos que su papel es de suma delicadeza y vital importancia, por lo cual es primordial que siempre prevalezca el hecho de que deba regirse por los principios de la ética profesional para Contadores, pues tratándose de aspectos tan fundamentales para las empresas y el sostenimiento social como lo son las finanzas, un contador que falte a estos principios, carece de confiabilidad y por lo tanto puede ser considerado como un mal elemento para el desarrollo de la actividad.

\section{Ley 43 de 1990}

Aprobada por el congreso de la republica el 13 de diciembre de 1990, en esta ley está contenida la regulación y principios de la ética profesional del contador público para ejercer sus facultades de la mejor manera y por la buena vía, para ser garantía de confiabilidad en las labores y facultades del contador público como profesional y su responsabilidad. "Los Contadores Públicos y las sociedades de Contadores Públicos quedan facultadas para contratar la prestación de servicios de las actividades relacionadas con la ciencia contable en general y tales servicios serán prestados por Contadores Públicos o bajo su responsabilidad" [6].

\section{¿Cuál es el papel del Contador Público?}

Ya identificado al Contador Público como el profesional que se encarga de la relación de establecer orden en lo financiero, dando opiniones basadas en la profesión para la toma de decisiones, estableciendo una gran responsabilidad en su facultad de actuar como asesor, para el mantenimiento del equilibrio en temas de vital importancia.

\section{La fe pública, el mayor de los compromisos}

Si existe una facultad de esta profesión que es notoria para el resto de la sociedad, es el poder validar la información contable y financiera por medio de la fe pública algo que es casi único de los contadores, pero al mismo tiempo esto acarrea una gran responsabilidad, pues cuando lo hacen esta dando por sentada y 


\section{2}

verídica esta información, se les otorga plena confianza de lo mismo.

\section{El Contador como actor social}

Previamente abarcados los conceptos básicos de los principales sujetos y factores que convocan a realizar el análisis y la posterior opinión sobre el tema, se conocio quien es el Contador Público, sus facultades, a que está sujeto el mismo para el correcto desarrollo de la profesión puesto que conlleva una gran responsabilidad lo que también genera un gran riesgo, en primera persona para el contador pero a su vez otros agentes se pueden ver afectados en múltiples aspectos, es justamente allí donde se presenta la conexión y relación directa entre la profesión contable y el resto de la sociedad, en Colombia el contador público se desempeña en 2 sectores, el privado y el público, los con alto impacto en la sociedad, "El Contador Público, sea en la actividad pública o privada es un factor de activa y directa intervención en la vida de los organismos públicos y privados. Su obligación es velar por los intereses económicos de la comunidad" [7].

Pues de ellos dependen muchas cosas que afectan a la misma dentro su funcionamiento habitual, lo cual se pueden poner en contexto con la labor contable, pues como lo se menciono en anteriores ocasiones el contador público tiene una alta relevancia en el funcionamiento, armonía y equilibrio de estos entes y a su vez de quienes dependen de los mismos y son parte de la misma sociedad. Además, también es considera un actor con responsabilidad social;

"El Contador Público, como profesional y responsable social, debe adquirir a gran escala principios y reglas de conducta anexos a la actividad contable, para aplicarlos en la ayuda y mejoramiento oportuno de la calidad de vida de la comunidad" [7]. Igualmente debe analizar pensamientos para la generación de ideas que contribuyan a su crecimiento interno, para la ampliación de su intelecto y así poder desarrollar una visión progresiva siempre con miras hacia el futuro, es decir generar una proyección, con el fin consolidar y mejorar los conceptos sociales para el mejoramiento del área contable.

Relación directa entre el contador, la ética y la sociedad

Ya habiendo recorrido los conceptos básicos, simples y compuestos de lo que comprenden cada uno de estos términos, se puede concluir que existe una relación directa entre cada uno de ellos y a continuación se identifican esos factores que conectan al Contador Público, la ética no es exclusiva de estos profesionales, ni otros, sino un patrón global que rige al ser humano para generar un equilibrio entre lo que se considera correcto, sin embargo esto es algo que debe pesar mucho sobre los hombros de un contador, debido a que ya se ha contextualizado lo importante y de cuidado que es el actuar de la profesión contable frente a los retos que enfrenta a diario, ya que ante una toma de una decisión considerada no correcta no solo él podría verse afectado, sino que se generaría talvez un efecto de cadena, donde se genere una afectación múltiple.

"Asimismo, los derechos también son importantes en la ética profesional, ya que si un profesional sostiene que hay algo que no responde a lo que él considera correcto en su pensamiento ético, no debería estar bajo ninguna circunstancia obligado a hacerlo" [8].

\section{Problemática de la ética contable}

En una sociedad como la colombiana seria excelente poder decir que priman los principios éticos en el desarrollo de cada individuo y particularmente en el tema de interés analizado en el presente articulo, en el actuar de los 
Contadores Públicos, sin embargo los hechos nos demuestran que existen múltiples casos de faltas a la ética por parte de contadores, y que han terminado afectando a otros agentes del entorno social, generando una problemática que perjudica por completo la profesión, perdiendo la confiablidad en la misma, todo esto se ve reflejado en un fenómeno social donde la afectación es global, solo por un impacto porcentual.

"Nos consideramos expertos en información financiera y en cuestiones fiscales y debemos pensar con toda seriedad en el problema que enfrentamos como profesionistas, al ejercer en un país que tiene el 3.5 de calificación -en la escala de 0 a 10-, y que ocupa el lugar 70 entre más de 160 países". [9]

Posibles amenazas para el compromiso ético y social

"Hoy en día nos encontramos con una sociedad degradada porque una gran mayoría de personas no atienden a las normas mínimas de convivencia y la falta de valores en muchos casos se hace evidente en el desempeño diario de nuestros profesionales en las diferentes áreas" [10]. Es aquí donde aparecen una serie de amenazas que impiden el cumplimiento del compromiso ético y se desvían hacia otras direcciones, que en la gran mayoría de ocasiones generan un daño en la integridad, no solo del profesional en manera individual, sino también de la profesión y de ello se pueden derivar consecuencias poco favorables.

\section{Desde la formación profesional}

La profesión contable ha venido sufriendo una serie deficiencias en materia de compromiso ético, pues cada vez son más los profesionales señalados de faltas a la ética en el ejercicio de la misma, esto se puede deber en cierta parte talvez a factores como, la formación profesional, el desconocimiento de varios aspectos referentes a la profesión, vacíos legales, incluso algunos fenómenos sociales.

\section{El desconocimiento}

Uno de los origines de la falta al compromiso de la ética profesional y no solo por parte del contador público, sino de muchos más se debe en parte a la poca formación ética que reciben durante su formación, pues la mención que se hace a esta, durante dicha etapa y en varias ocasiones casi que nula, por lo cual se pierde la esencia y se actúa de maneras poco correctas que en muchas ocasiones son causadas porque el profesional desconoce parcial o totalmente sus deberes y quien los regula

\section{La corrupción}

Cuando se menciona la palabra corrupción, es inevitable que se genere una preocupación por lo que este fenómeno produce en el ámbito social, puede producir un retroceso o un atraso en la misma, "La corrupción es sin lugar a dudas uno de los frenos que impiden el desarrollo de las potencialidades de Colombia como nación, incluye desde el mal uso del poder público para conseguir ventajas ilegitimas" [11].

Impacto social de las conductas antiéticas, es algo que se ha convertido en el pan de cada día de nuestra sociedad, hasta tanto que no hace falta quien trate de justificar estos comportamientos antiéticos, pero la verdad del asunto es que son actos que no deben tener ningún tipo de justificación puesto que casi el total de estos actos se cometen con plena conciencia, por lo tanto, se queda sin peso afirmar lo contrario, aun así hay un pequeño fenómeno que se puede presentar y es que estas cosas se puedan dar por lo enredadas y complejas que son las normas en Colombia, por tanto se pueda hacer una interpretación equivocada de la misma. 


\section{4}

"Un reciente informe de la Universidad Libre, revela que la raíz de la corrupción que ha salpicado la credibilidad y transparencia de algunos Contadores Públicos y revisores fiscales en el país, se debe, en parte, a la interpretación que estos profesionales hacen de las normas tributarias complejas, según su conveniencia e intereses, aprovechándose de su desconocimiento para hacerle 'conejo' a la ley" [12].

Aun así con lo anteriormente mencionado estas son eventualidades que se pueden presentar en el ejercicio de una actividad tan compleja como ha resultado ser la contaduría publica en un país como Colombia, un sin número de situaciones que derivan en este fenómeno que se podrían mencionar, peor que se conlcuye que lo relevante del asunto es el impacto que este produce, un impacto que también es social, pues se afecta a casi todos con esto, y también es una mala plataforma para lo que se ha venido analizando con respecto al compromiso ético, pues la corrupción produce desconfianza que es uno de los pilares de los cuales debe gozar un contador público, y a su vez termina afectando al toda la sociedad puesto que se ha convertido en un problema global que aunque ha estado latente por mucho tiempo, en la actualidad se ha vuelto tema de atención casi que en cualquier tema que se refiera a responsabilidad, poder y sociedad.

\section{Posibles soluciones}

Una posibles alternativas es afianzar el compromiso social del Contador Público en su actuar ético, pues ha quedado demostrado que sobre esto se ciernen muchas amenazas latentes que a diario generan unas consecuencias, por este motivo se suguieren algunos puntos importantes.
El Contador debe ser fortalecido en principios éticos desde su formación

Al ser la Contaduría un área tan delicada y compleja no se puede permitir que se falte a los principios éticos y se debe considerar como el mayor de los compromisos, la formación de un contador integral debe estar enfatizada desde los principios y no en un menor rango como a veces se suele manejar.

"El Contador debe ser una persona ética, con sentido de responsabilidad y honestidad, que mediante su trabajo no solo refleje compromiso con su entidad sino que también lo haga frente a su comunidad, dejando su profesión en lo más alto frente a los ojos de quienes son cercanos a ella" [13]. Por tanto, debemos considerar el comportamiento ético como el principal compromiso y de ahí derivaremos nuestro mejor desempeño como contadores, asesores y garantes de la verdad.

La ética del Contador como garante de la progresión social

Se debe tener en cuenta el concepto básico del contador público indica que es un profesional preparado para respaldar su actuar y su criterio, desenvolviéndose en asuntos que pueden representar mucha complejidad donde se afecta no solo èl como individuo sino también su entorno de acuerdo al impacto causado por este mismo, como se menciono en el párrafo anterior debe ser garante de la verdad y sinónimo de confianza, y esto genera un crecimiento o progresión con miras hacia su entorno, o lo que podríamos llamar sociedad.

\section{Conclusiones}

El análisis realizado sobre la importancia de la ética del contador público y que este debe tener como compromiso en el desempeño de sus labores, se establecio un contexto fuera de lo individual llevándolo hasta el ámbito 
social, donde se pudo analizar la relación que existe entre el contador y su compromiso con la sociedad basado en su buen actuar, se observo también los fenómenos y adversidades que se pueden presentar, como la falta de conocimiento, y el más terrible de todos, la corrupción que sin duda es el más temible puesto que se ha tomado muchas áreas profesionales, no solo la del contador público, ha permeado en la sociedad y parece ser un mal que no tiene fin, por todo esto y más se termina afectando el compromiso ético por parte del Contador Público.

Por otra parte también se exploro todos esos aspectos que construyen al profesional de la contaduría y publica en su ética profesional, y la relación que todo esto conlleva con los impactos en su entorno que se podría decir que se constituye como sociedad, pasando por lo legal como lo es la ley 43 de 1990 que regula el comportamiento y las facultades del contador público y los entes vigilantes y que lo validan como la JUNTA CENTRAL DE CONTADORES, por ultimo se sugerieron algunas posibles alternativas o soluciones que permitan la consolidación y el fortalecimiento de la conciencia y el sentido social en su labor como Contador Público garante.

\section{Referencias}

[1] N. Borja, "Perfil del Contador Público y su formación profesional hacia la adopción de los Estándares Internacionales del Profesional", Tesis de pregado, Universidad del Valle, 2012

[2] Congreso de La Republica de Colombia, "Ley 43”, Diciembre 1990. [En línea]. Disponible en: http://www.suin-juriscol.gov.co/viewDocument. asp?ruta $=$ Leyes $/ 1598256$

[3] J. Guevara, D. Giraldo, D. Gonzalez y H. Garcia, "Contribución del profesional contable para la sociedad", Septiembre 2015. [En línea]. Disponible en: https://www.gestiopolis.com/ contribucion-profesional-contable-sociedad/

[4] "La Prensa" Enero 2017. [En línea]. Disponible en: https://www.prensa.com/opinion/Eticaprofesional-trabajo_0_4666783335.html

[5] J.C. Martinez, "La Etica Profesional ¿Un problema del contador publico?", Revista Del Centro De Ciencias Investigativas $y$ Gerenciales, vol. 7, no. 1, pp. 74-89, 2010

[6] Congreso de La Republica de Colombia, Ley 43 de 1990, Bogota DC, 1990

[7] S. Betancur Gómez, M. Betancur Soto, N. García Ochoa y S. Jaramillo Gómez, "El poder de las organizaciones y la sociedad en el actuar del profesional contable", Agora, vol. 6, no. 6, pp. 93-104, 2005

[8] G. Roa, C. Martinez y J. Acosta, "Columbia. edu.py”, Noviembre 2017. [En línea]. Disponible en: https://www.columbia.edu.py/institucional/ revista-cientifica/articulos-de-revision/869-laetica-profesional

[9] C. Garcia Sabaté, Interviewee, Etica, defensa de una profesion. [Entrevista]. Mayo 2008

[10] D. Valencia Mosquera, "La ética profesional y el contador público en la actualidad", Marzo 2017. [En línea]. Disponible en:. https://www. ucc.edu.co/prensa/2016/Paginas/la-eticaprofesional-y-el-contador-publico-en-laactualidad.aspx

[11] C. Peña, "La Ética Profesional Como Base Para La Toma De Decisiones En La Gerencia Pública En Colombia", tesis de pregado, Universidad Militar, 2016

[12] "504 contadores públicos han sido sancionados por casos de corrupción, revela informe" Universidad Libre, 2017 


\section{6}

[13] C. Moncayo, "INPC", Diciembre 2015. [En línea]. Disponible en: www.incp.org.co

[14] "Niif.com", Diciembre 2019. [En línea]. Disponible en: https://niif.com.co/ley-43-1990/ codigo-de-etica-profesional

[15] “Mineducacion.gov.co", Julio 2005. [En línea]. Disponible en: https://www.mineducacion.gov. co/1621/article-85395.html 